
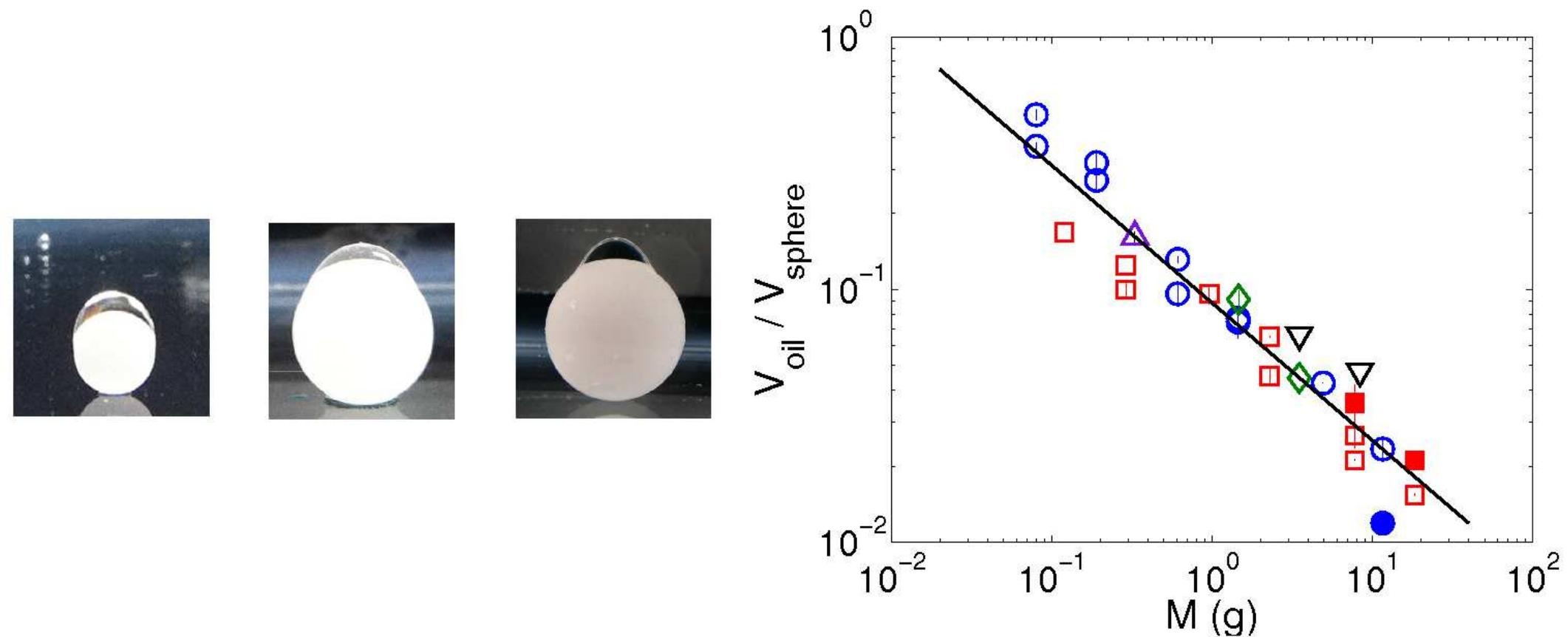

미 


\title{
Oil capture from a water surface by a falling sphere
}

\author{
Linda B. Smolka ${ }^{\mathrm{a}, *}$, Clare K. McLaughlin ${ }^{\mathrm{a}}$, Thomas P. Witelski ${ }^{\mathrm{b}}$ \\ ${ }^{a}$ Department of Mathematics, Bucknell University, Lewisburg, Pennsylvania 17837, USA \\ ${ }^{b}$ Department of Mathematics, Duke University, Box 90320, Durham, NC 27708, USA
}

\begin{abstract}
Motivated by contaminant remediation, we study the volume of oil (oleic acid) removed from a liquid lens by a falling particle. When a spherical particle is dropped from a fixed height into an oil lens that floats on top of a water surface, a portion of the oil adheres to the sphere. Once the sphere comes to rest at the subsurface, the oil forms an equilibrium pendant drop that remains attached to the sphere. We find in experiments with spheres of different sizes and materials, that the pendant drop volume is an increasing function of sphere mass for each material and a decreasing function of sphere density. By contrast, the normalized droplet volume in all of our experiments scales with sphere mass following $V_{\text {oil }} / V_{\text {sphere }} \sim M^{-0.544}$. Thus, for a given size, lighter spheres capture more oil relative to their own volume than do heavier spheres and are more efficient at removing oil from a water surface in our experiments. Estimates for the upper bound of the normalized droplet volume, determined from the continuous family of solutions of the Young-Laplace equation, show the same qualitative dependence on the sphere mass.
\end{abstract}

Keywords: Oil capture, Pendant drop, Drop shape, Interface, Young-Laplace Equation

\section{Introduction}

Contaminant remediation from an air-water interface is an active field of research whose interest was renewed after the 2010 Deepwater Horizon oil spill in the Gulf of Mexico. Studies have considered the role particles play in moving 5 contaminant from the surface to the subsurface by suspended particulate material residing in the fluid column [1 3], by dropping sand onto an oil spill (the sand-sink method) [4], and by granular rafts of oil drops encapsulated by sand particles [5].

In this experimental study, we consider a simple model problem to the sandsink method in which one spherical particle is dropped through an oil patch (or

\footnotetext{
* Corresponding author

Email addresses: lsmolka@bucknell.edu (Linda B. Smolka), witelski@math.duke.edu (Thomas P. Witelski)
}

Preprint submitted to Colloids and Surfaces A: Physicochemical and Engineering AspectsFebruary 1, 2016 

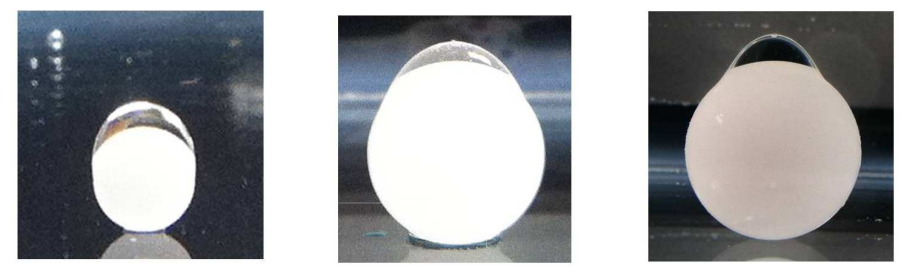

Figure 1: Pendant drops of oleic acid are attached to the top of delrin spheres. The spheres, which sit at the bottom of a tank of water, have radii (left-to-right): $R=0.238 \mathrm{~cm}, 0.476 \mathrm{~cm}$ and $0.953 \mathrm{~cm}$. The images are not at the same scale.

oil lens) that floats atop a water surface and address two fundamental questions: How effective is a single particle in removing oil from the water surface? Is there an optimal particle size that maximizes the capture of oil from the water surface? We examine particles of different sizes and materials in order to determine the key parameters that control oil entrainment by a sphere and to choose the particle size and material to optimize this. This model problem is analogous to sphere coating in which a sphere passes through an interface between two immiscible liquids of different viscosity [6] .

Approaches for using sand to capture oil from a water surface are more complicated than by a single spherical particle as it involves the sedimentation of a dispersion of particles [7 $[9]$. Numerous factors arise in sedimentation, including the dependence of the transport properties of a two-phase flow on the interactions between particles [10] and the dependence of the particle dynamics on the Reynolds number [11, 12]. Particle interactions are even more complicated in the presence of oil due to the capillary forces exerted between particles [13 16]. Determining the volume of oil captured is further complicated by the fact that collisions between particles can effect the number and size distribution of droplets attached to the particles [17] and that the interstitial fluid joining spheres varies with sphere configuration, orientation, radii and contact angle at

so the three-phase contact line [13]. For the simplified problem of a single particle, these issues do not arise and we will focus on analysis of the pendant drop volume attached to the particle when it comes to rest at the subsurface.

We find oil adheres to the sphere after it passes through the oil lens forming a static pendant drop attached to the top of the sphere once the sphere 35 comes to rest; examples are shown in Fig. 1. The shape of a pendant drop is governed by the Young-Laplace equation [18]. Solutions of the Young-Laplace equation compare well to experimental data for the quasi-static formation of drops and submerged bubbles on stationary planar, conical and spherical surfaces accurately predicting the profile and drop volume [19 25]. The Young-

40 Laplace equation has been extensively used to determine the interfacial tension and contact angle formed at a three-phase contact line of axisymmetric pendant drops at gas-liquid and liquid-liquid interfaces [18, 26 32]; methods include axisymmetric drop shape analysis [26, 28, 29] (ADSA) and a non-gradient based 
algorithm [30]. We examine the continuous family of solutions to the Young-

45 Laplace equation to determine theoretical upper bounds for the pendant drop volume and to assess the efficiency of oil capture by different particles.

The paper is organized as follows. In Section 2 we describe the experimental setup and procedures. In Section 3 we present experimental data for the pendant drop volume. In Section 4 we estimate upper bounds for the pendant drop

so volume from solutions of the Young-Laplace equation. Conclusions are provided in Section 5 .

\section{Experimental setup and procedure}

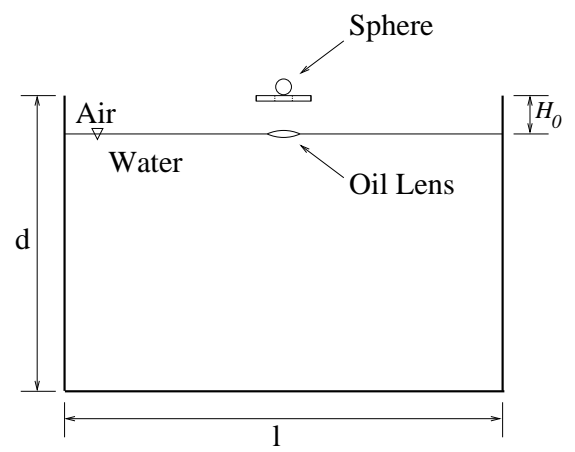

Figure 2: Schematic of experimental setup with an open top water tank, sphere and oil lens. The sphere is dropped from rest through a $1.2 \mathrm{~cm}$ thick sleeve to ensure vertical entry.

The experimental setup consists of an open top water tank, spheres and a hand-held digital camera. At the beginning of each experiment, the tank $55(\mathrm{l} \times \mathrm{w} \times \mathrm{d}=31 \mathrm{~cm} \times 15.7 \mathrm{~cm} \times 20.8 \mathrm{~cm})$ is washed with an industrial cleaner, dried and then filled $13 \mathrm{~cm}$ deep with tap water. For the majority of the experiments an oil lens of $350 \mu \mathrm{l}$ of oleic acid (90\%-grade Sigma-Aldrich, density $\rho_{o}=0.876 \mathrm{~g} / \mathrm{cm}^{3}$, surface tension $\gamma_{o}=32.5$ dynes $/ \mathrm{cm}$ at $20^{\circ} \mathrm{C}[33]$ ) is placed on the water surface $\left(\rho_{w}=1.0 \mathrm{~g} / \mathrm{cm}^{3}, \gamma_{w}=72.8\right.$ dynes $/ \mathrm{cm}$ at $20^{\circ} \mathrm{C}$

60 [33]). To ensure a sufficient supply of oil, a $500 \mu \mathrm{l}$ lens of oleic acid was also tested for the two largest spheres. Based on our experimental results (Section 3), we believe that the volume of the oil lens is sufficiently large so that it does not limit the volume of the pendant drop attached to the sphere. Oleic acid spreads on a water surface (with spreading coefficient $S_{o / w}=24.6 \mathrm{erg} / \mathrm{cm}^{2}$ at $20^{\circ} \mathrm{C}$

65 [34]) making it difficult to precisely control its size; in the experiments the lens radius ranged between $0.86-1.48 \mathrm{~cm}$. The room and water temperatures ranged between $22.6-24.1^{\circ} \mathrm{C}$ and $19.4-24.7^{\circ} \mathrm{C}$, respectively.

A schematic of the experimental setup is shown in Fig. 2. In the experiments, the oil lens is centered below the sphere's release point and the sphere is released

70 from rest a height $H_{0}=5.9 \mathrm{~cm}$ above the water surface entering the water with impact velocity $U_{0} \approx \sqrt{2 g H_{0}} \approx 1 \mathrm{~m} / \mathrm{s}$ which is below terminal velocity in air. 
Table 1: Spheres used in experiments: material, density and radii.

\begin{tabular}{lcc}
\hline Material & $\rho_{s}\left(\mathrm{~g} / \mathrm{cm}^{3}\right)$ & $R(\mathrm{~cm})$ \\
\hline delrin & 1.35 & $0.238,0.318,0.476,0.635,0.953,1.27$ \\
teflon & 2.13 & $0.238,0.318,0.476,0.635,0.953,1.27$ \\
glass & 2.41 & 0.318 \\
ceramic & 3.25 & $0.476,0.635$ \\
steel & 7.79 & $0.476,0.635$ \\
\hline
\end{tabular}

To ensure vertical entry, the sphere initially passes through a $1.2 \mathrm{~cm}$ thick sleeve. Each sphere is used only once.

Delrin, teflon, glass, ceramic and steel spheres (McMaster-Carr) ranging in 75 radius from $0.238-1.27 \mathrm{~cm}$ are examined in our study. Table 1 lists the densities and radii of the 17 different types of spheres used. The specifications for these spheres does not provide information on their wetting properties, so we will estimate the degree of wettability via analysis of experimental profiles to obtain contact angles.

Digital images are recorded of the sphere at rest at the tank bottom as shown in Fig. 1. The interface between the water and the oil and sphere is located from these images using edge-detection software in ImageJ and MATLAB.

\section{Experimental results}

Once the sphere is at rest at the bottom of the tank the entrained oil forms

${ }_{85}$ a pendant drop. The pendant drop is attached to the top of the sphere since the density difference between water and oleic acid is positive, causing buoyancy to generate an upward force on the droplet, away from the particle [18]. Three examples are shown in Fig.11for delrin spheres with radii $R=0.238 \mathrm{~cm}, 0.476 \mathrm{~cm}$ and $0.953 \mathrm{~cm}$ (left-to-right) where both the interfacial shape, including the three-

90 phase contact line between the oil, water and sphere, and volume of the pendant drop are in equilibrium.

Figure 3 shows examples from experiments of pendant drops attached to (a) delrin, (b) teflon and (c) glass spheres, respectively, where solid lines represent the interface between the pendant oil drops attached to the spheres and water 95 and dashed lines represent the surface of the spherical particles. We observe that drop shapes vary significantly according to the wetting properties of the sphere and also according to the size of the sphere.

The volume of the pendant drop is computed from the experimental data for the interface shape, $X(Z)$ (see Fig. 3). Assuming axisymmetry,

$$
V_{\mathrm{oil}}=\pi \int_{Z_{c}}^{Z_{0}}[X(Z)]^{2} d Z-\pi \int_{Z_{c}}^{2 R}\left[R^{2}-(Z-R)^{2}\right] d Z,
$$

where $Z_{c}$ and $Z_{0}$ are the vertical positions of the three-phase contact line and drop apex, respectively, and the sphere occupies the range $0 \leq Z \leq 2 R$. The

first integral in Eq. (11) is the combined sphere and pendant drop volume and 

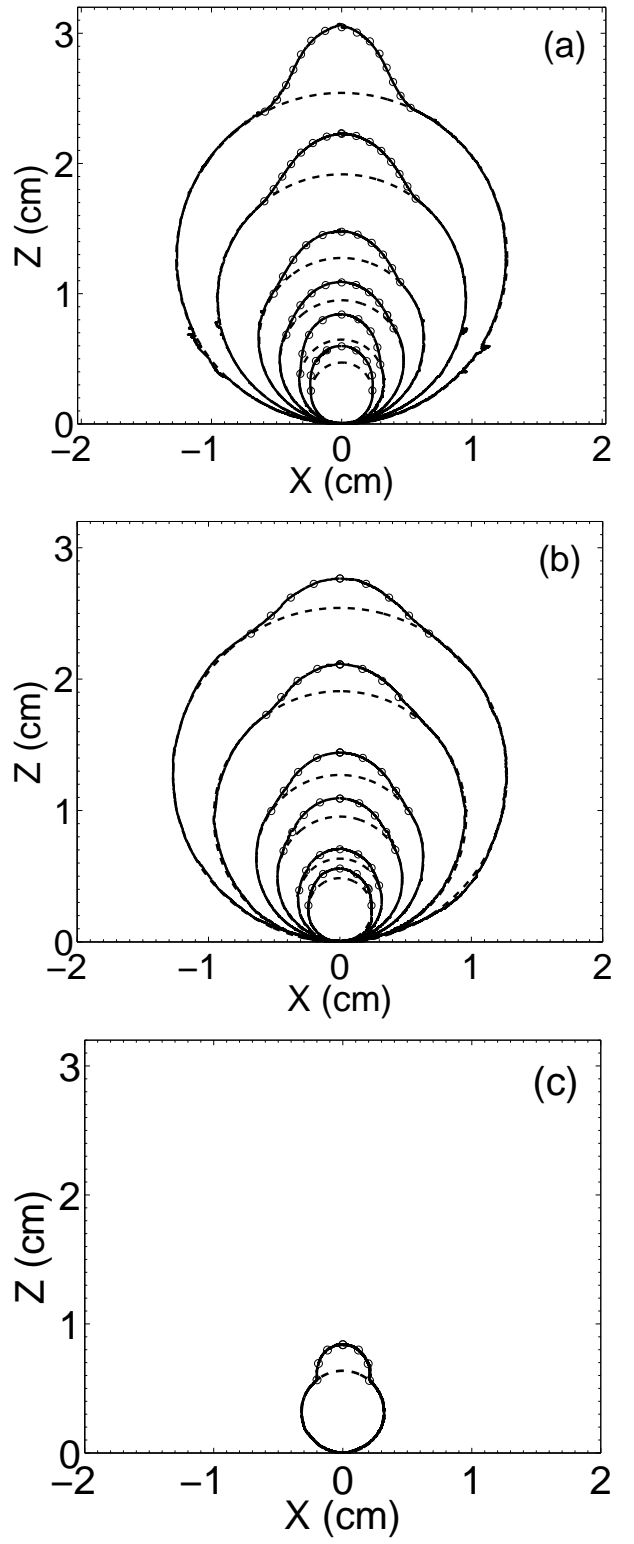

Figure 3: Digitized profiles of pendant oil drops attached to spheres that are at rest on the bottom of a water tank (solid lines). The pendant drops are in equilibrium. (a) Overlay of six delrin spheres; (b) overlay of six teflon spheres; and (c) a glass sphere; the sphere radii are listed in Table 1 Dashed lines represent the surface of each spherical particle. Circles denote the best fit numerical solutions of the Young-Laplace equation (4). 


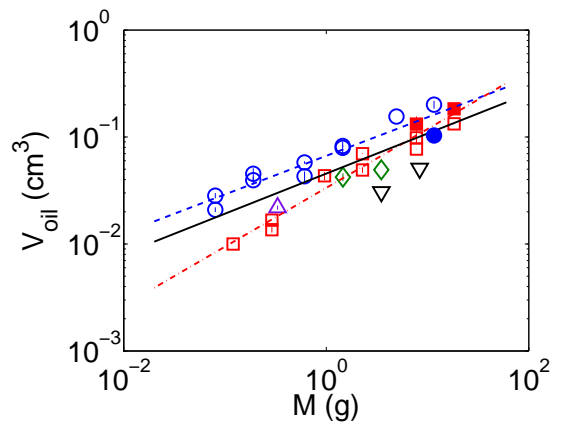

Figure 4: Pendant drop volume as a function of sphere mass. Best fits: All spheres $-V_{\text {oil }}=$ $0.05 M^{0.375}$ (solid line); delrin spheres - $V_{\text {oil }}=0.07 M^{0.361}$ (dashed line); teflon spheres $V_{\text {oil }}=0.03 M^{0.550}$ (dot-dashed line). Symbols: delrin $(\circ)$, teflon $(\square)$, glass $(\triangle)$, ceramic $(\diamond)$, steel $(\nabla), 350 \mu \mathrm{l}$ oleic acid lens (open), $500 \mu \mathrm{l}$ oleic acid lens (filled).

the second integral is the volume of the spherical cap where both integrals are measured above the contact line. The oil volume is calculated on the left and right-half of each pendant drop; ideally in the axisymmetric case these should be equal. Figure 4 shows the volume as a function of sphere mass with the vertical bars representing the left and right-half volumes and the symbols their mean value; the vertical bars are very short and may not be visible on the scale of the figure indicating that the drops are very close to axisymmetric. The asymmetric effects are relatively small as seen in the profiles from Fig. 3. The pendant drop volume increases with sphere mass and also with sphere radius for a particular material with the results independent of the oil lens volume placed at the water surface. The pendant drop volume also depends on the sphere density, decreasing as density increases. In previous experiments on spheres rising through a water-silicone oil interface, the volume of water entrained by the sphere increases with the sphere volume possibly scaling with the sphere's terminal velocity in water which increases as the sphere volume increases [35]. Although this trend is analogous to our data since $V_{\text {sphere }} \sim M$, the dynamics in our experiments are different given that the sphere entry velocity $U_{0}$ is fixed.

To measure the efficiency of oil capture, we consider the normalized pendant drop volume $V_{\text {oil }} / V_{\text {sphere }}$ which measures the amount of oil a particle entrains relative to its own volume. Figure 5 shows that the normalized droplet volume is a decreasing function of the sphere mass with all of our experimental data collapsing to the power-law

$$
V_{\text {oil }} / V_{\text {sphere }} \sim 1 / M^{0.544},
$$

indicating that efficiency of oil removal increases as the mass decreases. Hence relative to their own volume, a lighter sphere entrains a greater volume of oil 120 than does a heavier sphere. This relation has at most a weak dependence on the material properties of the spheres used in our study. There is a critical value of $M$ for our experimental conditions below which the particle's weight will not 


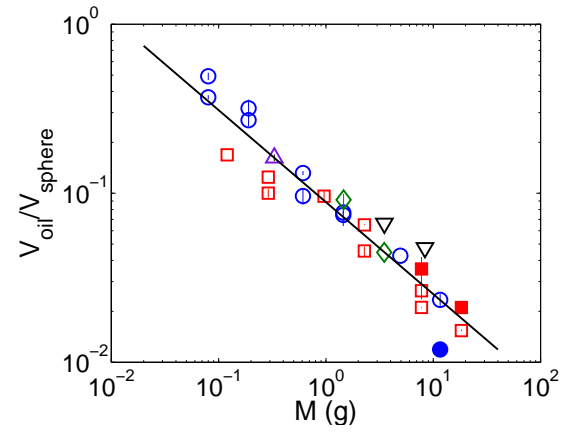

Figure 5: Normalized droplet volume $V_{\text {oil }} / V_{\text {sphere }}$ as a function of sphere mass. Solid line is a best fit of all of the data, $V_{\text {oil }} / V_{\text {sphere }}=0.089 / M^{0.544}(\mathrm{CC}=0.90)$. Symbols: delrin $(\circ)$, teflon $(\square)$, glass $(\triangle)$, ceramic $(\diamond)$, steel $(\nabla), 350 \mu \mathrm{l}$ oleic acid lens (open), $500 \mu \mathrm{l}$ oleic acid lens (filled).

overcome the interfacial tension of the fluids to penetrate the free surface, hence the validity of the power-law scaling (2) must have a lower bound on $M$.

\section{Drop Analysis}

One may wonder whether the maximum volume of oil that a sphere can capture occurs in our experiments. The amount of oil captured by a sphere must be controlled by the transient hydrodynamics of the particle passing through the oil lens and settling through the fluid column to the subsurface and answering this question requires further work beyond this study. We can, however, obtain upper bounds for the pendant drop volume by examining solutions of the Young-Laplace equation. We begin by introducing the Young-Laplace equation and demonstrating that best fit solutions of the Young-Laplace equation are in excellent agreement with the pendant drop profiles in our experiments before determining theoretical bounds for the pendant drop volume.

The profile of the pendant drop located at the oleic acid and water interface, as shown in Fig. [6, is governed by the Young-Laplace equation [18]

$$
\gamma_{w o}\left(\frac{d \phi}{d s}+\frac{\sin \phi}{x}\right)=\frac{2 \gamma_{w o}}{R_{0}}+z \triangle \rho g,
$$

where $\gamma_{w o}$ is the interfacial tension of oleic acid against water, $R_{0}$ is the radius of curvature at the apex of the drop, $\triangle \rho=\rho_{w}-\rho_{o}, g$ is acceleration due to gravity, $s$ denotes arclength, and $\phi$ is measured from the horizontal to the tangent to the pendant drop. The terms on the left-hand side of Eq. (3) are the sum of the principal radii of curvature of the pendant drop and the terms on the right represent the excess pressure inside the surface at the apex and the hydrostatic pressure difference over height $z$. The Young-Laplace equation (3) modeling the pendant drop in Fig. 6 can be expressed as a system of ordinary differential 


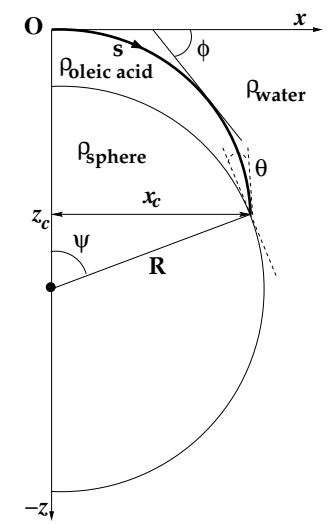

Figure 6: Schematic of an axisymmetric pendant drop attached to the top of a solid sphere of radius $R$.

equations in standard form [28, 29]

$$
\begin{aligned}
\frac{d \phi}{d s} & =\frac{2}{R_{0}}-\frac{\sin \phi}{x}+c z, \\
\frac{d x}{d s} & =\cos \phi, \\
\frac{d z}{d s} & =-\sin \phi,
\end{aligned}
$$

satisfying the boundary conditions at the apex

$$
\phi(0)=x(0)=z(0)=0,
$$

145 where $c=\triangle \rho g / \gamma_{w o}=\ell_{c}^{-2}$ is a capillary constant which can be expressed in terms of the capillary length $\ell_{c}$. We orient the $z$-axis in the opposite direction to [28, 29] which changes the sign in Eq. (4c) compared to their system. The experimental coordinates $(X, Z)$ in Fig. 3 are related to $(x, z)$ by $(X, Z)=$ $\left(x, z+Z_{0}\right)$. The contact line occurs where the drop profile intersects the surface 150 of the sphere, at $\left(x_{c}, z_{c}\right)$ satisfying $x_{c}^{2}+\left(Z_{0}-R+z_{c}\right)^{2}=R^{2}$, as shown in Fig. 6. The parameters in this boundary value problem are $R_{0}, c$ and $Z_{0}$.

Following Smith and van de Ven 21], the solution of the boundary value problem can be used to determine the filling angle of the pendant drop,

$$
\psi=\sin ^{-1}\left(\frac{x_{c}}{R}\right),
$$

and the contact angle,

$$
\theta=\phi_{c}-\psi,
$$

where $\phi_{c}$ is measured to the three-phase contact line.

Since analytic solutions to the boundary value problem (4) are not available, we compute solutions numerically using a fourth-order Runge-Kutta method. 
We use a simpler approach than ADSA [26, 28, 29] or the non-gradient method [30] to determine the best fit theoretical curves to the experimental profiles since we are unable to obtain the image quality required by these methods in our experimental setup.

Given $N$ data points $\left(X_{n}, Z_{n}\right)$ in the experimental profile, we use linear interpolation of the computed solution to estimate the solution at $X_{n}$ denoted by $\left(X_{n}, z\left(X_{n}\right)\right)$ or at $Z_{n}$ denoted by $\left(x\left(Z_{n}\right), Z_{n}\right)$. The best fit values of $R_{0}, c$ and $Z_{0}$ are determined by minimizing either the squared vertical distances summed over the $N$ points

$$
D_{z}=\sum_{i=1}^{N}\left[z\left(X_{n}\right)-Z_{n}\right]^{2},
$$

or the squared horizontal distances

$$
D_{x}=\sum_{i=1}^{N}\left[x\left(Z_{n}\right)-X_{n}\right]^{2},
$$

between the estimated Young-Laplace solutions and the experimental profile.

160 The numerical method to estimate the parameters consists of a Monte Carlo optimization process for the solutions of system (4) that minimize $D_{z}$ or $D_{x}$. For each experimental profile between 20,000 and 60,000 numerical simulations were run with random values of the parameters $R_{0}, c$ and $Z_{0}$. Examining the resulting distribution of values for $D_{z}$ or $D_{x}$ suggested that near optimal solutions have been found. $D_{x}$ is a better measure than $D_{z}$ when the pendant drop profile is nearly vertical at the three-phase contact line as for the smallest spheres. For the other spheres, we find the best fit parameters $R_{0}, c$ and $Z_{0}$ are nearly the same when minimizing either $D_{z}$ or $D_{x}$. Huh and Reed [32] use a different approach to obtaining the best fit Young-Laplace solutions for droplet data 170 profiles but comment on the differences in weighting of data in using $D_{z}$ vs. $D_{x}$. For asymmetric pendant drops, best fit curves are computed on the left and right-half of the pendant drop profile.

Figure 3 shows that the best fit Young-Laplace solutions, denoted by circles, are in excellent agreement with the experimental profiles. The best fit solution 175 characterizes the pendant drop shape from which we obtain estimates for the radius of curvature at the apex and the filling and contact angles. The best fit solution also aids in estimating the interfacial tension of oleic acid against water via the capillary constant. The values of $R_{0}, \psi, \theta, c$ and $\gamma_{w o}$ for all of the experiments are provided in the Appendix.

180 We expect that the volume of oil captured by a falling sphere has some dependence on the transient hydrodynamics of the sphere settling though the fluid column to the subsurface. Furthermore, under different hydrodynamic conditions we expect that either more or less oil could be captured. We anticipate that the capillary parameter and the contact angle are fixed while the features 185 of the pendant drop shape $\left(R_{0}\right.$ and $\left.Z_{0}\right)$ can vary to allow for a range of volumes.

There is a continuous family of solutions to the Young-Laplace equation (4) over a range of apex heights $Z_{0}$ with $c$ and $\theta$ held fixed that can be used to obtain 

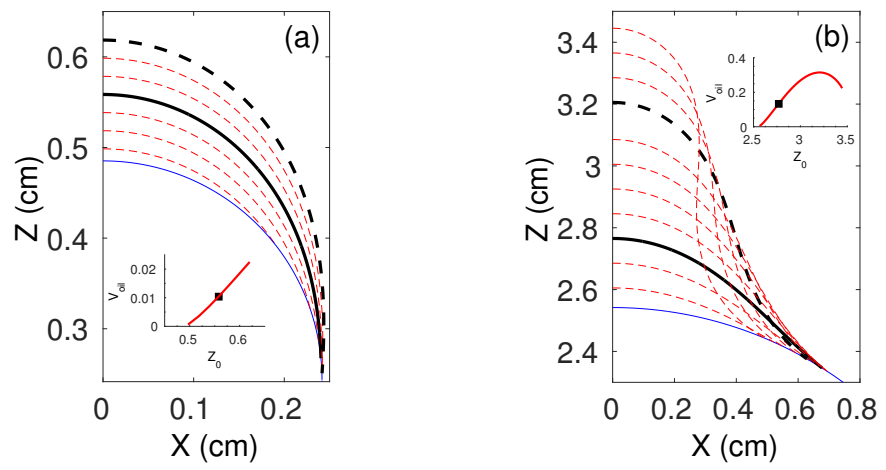

Figure 7: Solutions of the Young-Laplace equation for the pendant drop profile attached to teflon spheres plotted over a range of apex heights with: (a) $R=0.238 \mathrm{~cm}$ and (b) $R=1.27 \mathrm{~cm}$. Parameters, selected from the best fit Young-Laplace solution to experimental data, are: (a) $c=11.78 \mathrm{~cm}^{-2}, \theta=4.10^{\circ}$ and (b) $c=9.51 \mathrm{~cm}^{-2}, \theta=6.15^{\circ}$. Thin solid lines represent the sphere surface, thick solid lines represent the best fit theoretical curve to the observed experimental profiles and thick dashed lines are the theoretical profiles with the largest pendant drop volume. Each subplot shows volume $\left(\mathrm{cm}^{3}\right)$ as a function of the height of the apex of the pendant drop $(\mathrm{cm})$ for the theoretical profile with the square indicating the volume observed in the experiment.

both a theoretical range and upper bound for the pendant drop volume; values of $c$ and $\theta$ are taken from the best fits to the experimental data. Examples

are shown in Fig. 7 for the smallest and largest teflon spheres used in our experiments where the thick solid lines represent the best fit solutions to the experimental data. Note that the best fits for plots (a) and (b) resulted in different values for $c$; these values are within the standard deviation of measured values as reported in the Appendix. Not all of these solutions may be stable, particularly those that exhibit an overturn in the profile as in Fig. 7 (b). The subplots show the drop volume as a function of the apex height, $Z_{0}$, for each family of solutions where squares denote the experimental value. The droplet profile with the maximum volume (not necessarily the one with the largest $Z_{0}$ ) are indicated by the thick dashed lines. In both of the experiments shown here, it is clear that there is a significant difference between the maximum-volume solution and the experimental profile. In similar analysis, we find that the maximum pendant drop volume is not attained by any of the spheres used in our study. Although it is not clear if the droplet profiles corresponding to the maximum volumes are stable, they do provide an upper bound on the volume of the pendant drop in each case. It is worth noting that theoretical studies predict stable axially symmetric pendant drops hanging from a planar surface must have volume be an increasing function of the drop height [36, 37]. If the same holds true for the pendant drops in our experiments, then the solutions above the thick dashed solution in Fig. 7(b) would be unstable. Analyzing the range of particle sizes in our study, we find that the pendant drop volume either increases monotonically with the drop height as in Fig. 7(a) or attains just one 


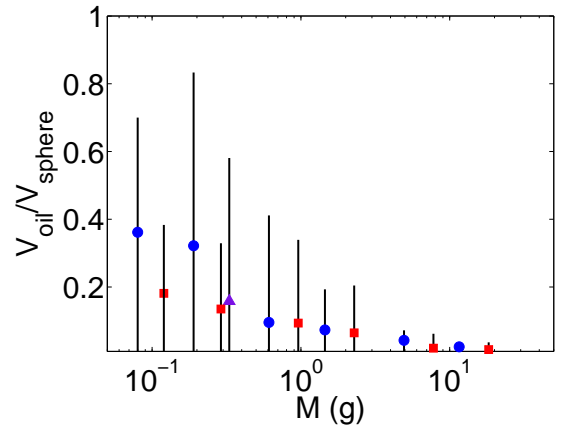

Figure 8: Normalized droplet volume as a function of sphere mass for delrin (•), teflon ( and glass $(\boldsymbol{\Delta})$ spheres. Symbols represent the normalized volume of pendant drops observed in the experiments. Vertical bars indicate the range of the normalized droplet volume computed from the corresponding family of solutions to the Young-Laplace equation with the parameters $c$ and $\theta$ for each sphere taken from the best fit solution to the experimental data.

maxima as in Fig. 7(b).

The data in each of the subplots of Fig. 7 provides a theoretical range for the pendant drop volume that can attach to each of the teflon spheres; for the smaller sphere $0 \leq V_{\text {oil }} \leq 0.022 \mathrm{~cm}^{3}$ and for the larger sphere $0 \leq V_{\text {oil }} \leq$ $0.315 \mathrm{~cm}^{3}$. Normalizing this data by the corresponding sphere volume yields the theoretical range for the normalized droplet volume; for the smaller sphere $0 \leq V_{\text {oil }} / V_{\text {sphere }} \leq 0.390$ and for the larger sphere $0 \leq V_{\text {oil }} / V_{\text {sphere }} \leq 0.037$. Figure 8 shows the range of normalized droplet volume denoted by vertical bars 220 for the delrin $(\bullet)$, teflon ( $\boldsymbol{\square})$ and glass ( $\mathbf{\Delta})$ spheres used in our study. The data is plotted versus the sphere mass and the symbols denote the experimentally measured values. We showed in Fig. 5 that the normalized droplet volume of the spheres observed in our experiments decreases with mass. We find the same general trend with the theoretical range, though we note that the stable range of normalized droplet volumes may be less than those shown in Fig. 8 based upon the stability of the family of solutions to the Young-Laplace equation. Nevertheless, Fig. [8] shows that lighter spheres are the most efficient at removing oil from the water surface in the idealized case that each sphere captures the maximum volume of oil predicted from theory.

\section{Conclusions}

Motivated by contaminant remediation by the sand-sink method, we have examined the volume of oil removed from a liquid lens by a falling particle. When a spherical particle is dropped from a fixed height into an oil lens of oleic acid that floats on top of a water surface, a portion of the oil adheres 235 to the sphere. After the sphere comes to rest at the subsurface, the oil forms an equilibrium pendant drop that remains attached to the sphere effectively removing oil from the water surface. 
The volume of oil captured by a sphere increases with the sphere's mass, analogous to the volume of water drawn into a layer of silicone oil by a rising sphere in a two-layer stratified fluid 35]. However, we find from experimental data and an analysis of the continuous family of solutions of the Young-Laplace equation that lighter spheres capture more oil relative to their own volume than do heavier spheres; this trend has at most a weak dependence on the material properties of the spheres considered in our study. Consequently, lighter parti-

245 cles are the most efficient at removing oil from a water surface. This provides some evidence that sand is a good choice for remediating oil spills. Definitive proof requires a detailed investigation of a dispersion of particles which would complement the existing research on the effectiveness of suspended particulate materials in removing oil from a sea surface [1-3].

The scaling of the normalized droplet volume found in our experiments Eq. (2) is equivalent to $V_{\text {oil }} / V_{\text {sphere }} \sim R^{-1.63}$ which is quite different from the scaling found in the analogous problem of sphere coating $V_{\text {oil }} / V_{\text {sphere }} \sim \ln R$ [6] (holding the interfacial tension and sphere and liquid densities fixed). In the coating problem the sphere passes through a stratification interface from a

255 highly viscous upper liquid into water. In our study the sphere free falls through air before passing through a thin liquid lens and into water. This difference in scalings highlights the importance of the thin oil layer configuration.

We believe the scalings in our idealized study hold for low velocity entry by a sphere initially free falling through air. This study provide some evidence on the efficiency with which particles can remove oil from a free surface. The scalings, however, may break down at high velocity entry, depend on the depth of the fluid column above the subsurface and may change with the type and polarity of the oil, all matters left for future work. The scaling that we have found for the normalized droplet volume also motivates the need for further direct numerical

265 simulations and modeling studies to explain how different hydrodynamic effects and material properties might interact to yield the dependence observed in this study.

\section{Acknowledgements}

We are grateful to Moises Veloz for his help in building the experimental 270 apparatus and we mourn his passing. We wish to acknowledge the support of the National Science Foundation Grant Nos. DMS-0707755 and DMS-0968252.

\section{Appendix A. Parameter values for best fit solutions of the Young- Laplace equation}

Best fit solutions of the Young-Laplace equation are made to the pendant 275 drop profiles. From these best fits, the radius of curvature at the apex $\left(R_{0}\right)$ and the filling and contact angles ( $\psi$ and $\theta$, respectively) of the pendant drop are determined for each particle used in our experiments; values of $R_{0}, \psi$ and $\theta$ are shown in Fig. A1(a), (b) and (c), respectively. Excluding the glass sphere, 

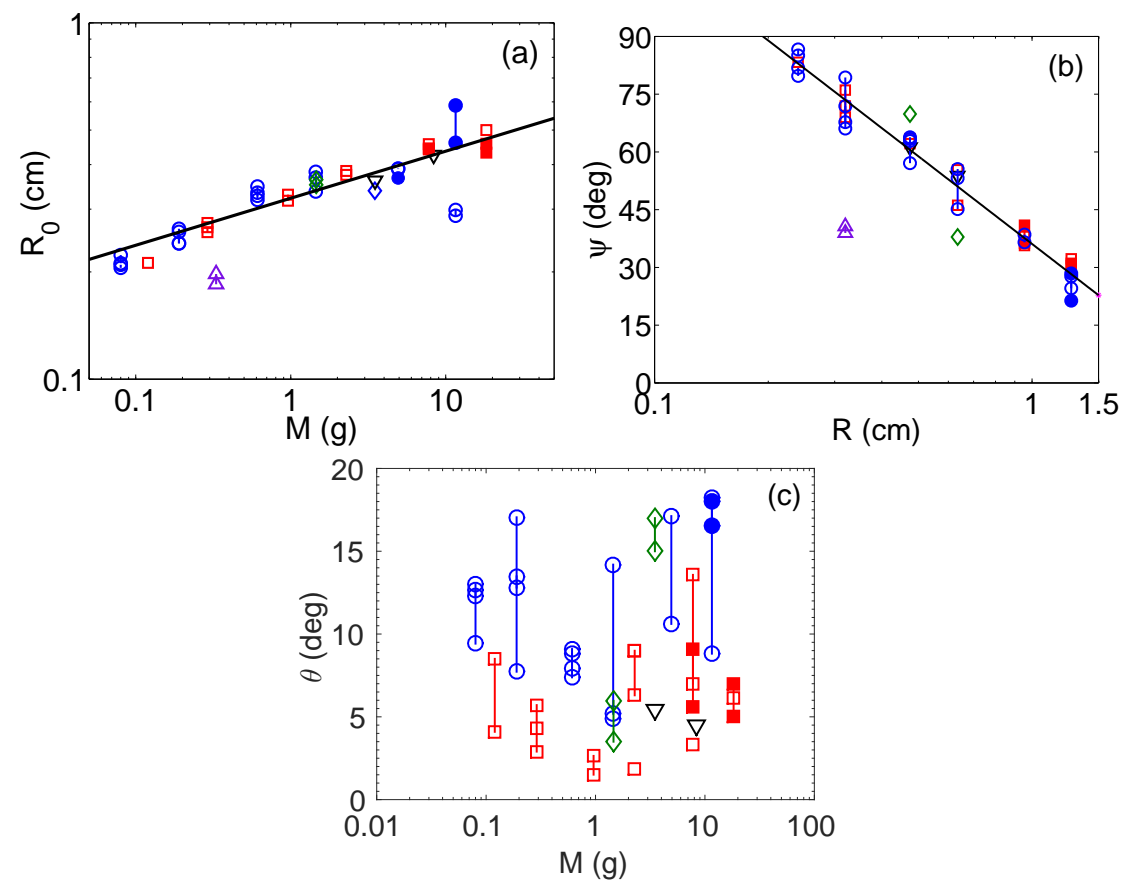

Figure A1: (a) Radius of curvature of the apex of the pendant drop as a function of sphere mass. Solid line is a best fit of the data excluding the glass sphere, $R_{0}=0.322 M^{0.132}$ $(\mathrm{CC}=0.87)(\mathrm{b})$ Filling angle of the pendant drop as a function of sphere radius. Solid line is a best fit of the data excluding the glass sphere, $\psi=-75.6 \log (R)+36.1(\mathrm{CC}=0.97)$. (c) Contact angle at the three-phase contact line as a function of sphere mass. Average values of contact angle: $\theta_{\text {steel }}=5.0^{\circ}, \theta_{\text {teflon }}=5.4^{\circ}, \theta_{\text {ceramic }}=10.4^{\circ}, \theta_{\text {delrin }}=11.4^{\circ}, \theta_{\text {glass }}=57.4^{\circ}$ (not shown). Symbols: delrin $(\circ)$, teflon $(\square)$, glass $(\triangle)$, ceramic $(\diamond)$, steel $(\nabla), 350 \mu \mathrm{l}$ oleic acid lens (open), $500 \mu \mathrm{l}$ oleic acid lens (filled). The left and right-half values for asymmetric pendant drops are joined by vertical lines.

we find that the radius of curvature increases with mass following the scaling $R_{0} \sim M^{0.132}$ and the filling angle decreases logarithmically with increasing sphere radius with the pendant drop nearly covering the upper hemisphere of the smallest delrin and teflon spheres. The contact angle for each material is nearly constant within the scatter of the data due to asymmetry of the pendant drop in some experiments with steel $\left(\theta=5.0^{\circ}\right)$ and teflon $\left(\theta=5.4^{\circ}\right)$ having ( $\left(\theta=10.4^{\circ}\right)$ and delrin $\left(\theta=11.4^{\circ}\right)$ having moderate values and glass $\left(\theta=57.4^{\circ}\right)$ the highest value (glass data not shown in figure).

The best fit values of the capillary constant, $c$, exhibit scatter similar to the measurements of the contact angle. The mean value of $c$ was found to be $10.1 \mathrm{~cm}^{-2}$ with a standard deviation of $\pm 1.88 \mathrm{~cm}^{-2}$. This corresponds to a mean value of the interfacial tension of oleic acid against water, $\bar{\gamma}_{w o}=12.1$ dynes $/ \mathrm{cm}$. The range of the interfacial tension within one standard deviation of $c$ is $10.2 \leq$ 
$\gamma_{w o} \leq 14.8$ dynes/cm. Image quality has a significant impact on measurements of surface tension using solutions of the Young-Laplace equation [29] which explains the scatter exhibited in $\gamma_{w o}$, and for that matter $\theta$, in our study. We note, however, that the range of $\gamma_{w o}$ is not far from the expected values given that $\gamma_{w o}=15.7$ dynes $/ \mathrm{cm}$ at $20^{\circ} \mathrm{C}$ (following the relation $\gamma_{w o}=\gamma_{w}-\gamma_{o}-$ $S_{o / w}\left[\begin{array}{ll}34\end{array}\right)$ and that the temperature in our experiments exceeded $20^{\circ} \mathrm{C}(\gamma$ is a decreasing function of temperature).

[1] D. K. Muschenheim, K. Lee, Removal of oil from the sea surface through particulate interactions: review and prospectus, Spill Sci. Technol. Bull. 8 (2002) 9-18.

[2] J. R. Payne, J. R. Clayton, B. E. Kirstein, Oil/suspended particulate material interactions and sedimentation, Spill Sci. Technol. Bull. 8 (2003) 201221.

[3] L. Sorenson, A. G. Melbye, A. M. Booth, Oil droplet interaction with suspended sediment in the seawater column: influence of physical parameters and chemical dispersants, Mar. Pollut. Bull. 78 (2014) 146-152.

[4] R. A. A. Blackman, Toxicity of oil sinking agents, Mar. Pollut. Bull. 5 (1974) 116-118.

[5] M. Abkarian, S. Protiere, J. M. Aristoff, H. A. Stone, Gravity-induced encapsulation of liquids by destabilization of granular rafts, Nat. Commun. 4 (2013) 1895.

[6] O. Pitois, P. Moucheront, C. Weill, Interface breakthrough and sphere coating, C. R. Acad. Sci. Paris IIB 327 (1999) 605-611.

[7] A. D. Maude, R. L. Whitmore, A generalized theory of sedimentation, Br. J. Appl. Phys. 9 (1958) 477-482.

[8] G. K. Batchelor, Sedimentation in a dilute dispersion of spheres, J. Fluid Mech. 52 (1972) 245-268.

${ }_{320}[9]$ R. H. Davis, A. Acrivos, Sedimentation of noncolloidal particles at low Reynolds numbers, Ann. Rev. Fluid Mech. 17 (1985) 91-118.

[10] G. K. Batchelor, Transport properties of two-phase materials with random structure, Ann. Rev. Fluid Mech. 6 (1974) 227-255.

[11] E. Guazzelli, J. Hinch, Fluctuations and instability in sedimentation, Ann. Rev. Fluid Mech. 43 (2011) 97-116.

[12] A. F. Fortes, D. D. Joseph, T. S. Lundgren, Nonlinear mechanics of fluidization of beds of spherical particles, J. Fluid Mech. 177 (1987) 467-483.

[13] E. Bayramli, A. Abou-Obeid, T. G. M. van de Ven, Liquid bridges between spheres in a gravitational field, J. Colloid Interface Sci. 116 (1987) 490-502. 
[14] E. Bayramli, T. G. M. van de Ven, An experimental study of liquid bridges between spheres in a gravitational field, J. Colloid Interface Sci. 116 (1987) 503-510.

[15] R. K. Niven, N. Khalili, D. B. Hibbert, Mixed solid/dispersed phase particles in multiphase fluidised beds, Part I: Free energy of stability due to interfacial tension, Chem. Eng. Sci. 55 (2000) 3013-3032.

[16] R. K. Niven, N. Khalili, D. B. Hibbert, Mixed solid/dispersed phase particles in multiphase fluidised beds. II: Stability at laminar to turbulent flow scales, Chem. Eng. Sci. 55 (2000) 3033-3051.

[17] T. Dabros, T. G. M. van de Ven, Collision-induced dispersion of droplets attached to solid particles, J. Colloid Interface Sci. 163 (1994) 28-36.

[18] S. Hartland, R. W. Hartley, Axisymmetric Fluid-Liquid Interfaces, Elsevier, 1976.

[19] J. E. Halligan, L. E. Burkhart, The profile of a separating droplet, J. Colloid Interface Sci. 27 (1968) 127-132.

[20] E. Shoukry, M. Hafez, S. Hartland, Separation of drops from wetted surfaces, J. Colloid Interface Sci. 53 (1975) 261-270.

[21] P. G. Smith, T. G. M. van de Ven, The separation of a liquid drop from a stationary solid sphere in a gravitational field, J. Colloid Interface Sci. 105 (1985) 7-20.

[22] K. Katoh, H. Iyota, T. Inoue, T. Tsujino, Volume of a liquid drop detaching from a sphere, Heat Transfer - Asian Research 39 (2010) 396-409.

[23] S. R. Babu, Analysis of drop formation at conical tips. 1. Theory, J. Colloid Interface Sci. 116 (1987) 350-358.

[24] S. R. Babu, Analysis of drop formation at conical tips. 2. Experimental, J. Colloid Interface Sci. 116 (1987) 359-372.

[25] D. Gerlach, G. Biswas, F. Durst, V. Kolobaric, Quasi-static bubble formation on submerged orifices, Int. J. of Heat and Mass Transfer 48 (2005) 425-438.

[26] Y. Rotenberg, L. Boruvka, A. W. Neumann, Determination of surface tension and contact angle from the shapes of axisymmetric fluid interfaces, J. Colloid Interface Sci. 93 (1983) 169-183.

[27] D. B. Thiessen, D. J. Chione, C. B. McCreary, W. B. Krantz, Robust digital image analysis of pendant drop shapes, J. Colloid Interface Sci. 177 (1996) 658-665. 
[36] E. Pitts, The stability of pendent liquid drops. Part 2. Axial symmetry, J. Fluid Mech. 63 (1974) 487-508.

[37] H. C. Wente, The stability of the axially symmetric pendent drop, Pac. J. Math. 88 (1980) 421-470. 